\title{
Research on the Translation Teaching Exploration Based on the Parallel Corpus
}

\author{
Ping Chen ${ }^{1, a}$ \\ ${ }^{1}$ Department of Foreign Languages, Ankang University, Shaanxi 725000, China \\ achenpingak@163.com
}

Keywords: Parallel Corpus, Translation Teaching, Exploration

\begin{abstract}
Parallel corpus got attention and was widely established in the 1990s, it has great significance for comparative study, translation teaching which include automatic translation and bilingual of dictionary compilation. The application of parallel corpus in translation teaching is mainly reflected at three levels, namely the vocabulary, sentence and discourse.
\end{abstract}

\section{Introduction}

At present, bilingual or multilingual parallel corpus linguistics corpus is becoming an important direction. Parallel corpus is to synchronize two or more languages for translation on interactive corpus paragraph, sentence or even word level and there are the original corpus, additional code corpus and parallel corpus and grid-style corpus types. Given the typical language features on the corpus of words, sentences and paragraphs chapter, on the basis of parallel corpora development fact sheets on from words, sentences and discourse analysis to explore three levels parallel corpus application in Translation Teaching [1]

As an emerging field of teaching and research, based translation teaching Corpus academic attention is increasingly being Combining Corpus and Translation Teaching is an innovation in the field of translation teaching. As noted by Murray learner teaching environment in the corpus can take "discovery learning and data-driven approach to learning to master translation skills training, improve translation skills, familiar with translation occupational characteristics" by the management and operation of the corpus to reform the traditional translation teaching mode, the translation industry has become the consensus of many scholars.

\section{The Establishment and Role of Parallel Corpus}

The Establishment of Parallel Corpus. Since the 1960s, Brown University (Brown University) linguist W. Nelson Francis and Henry Kucera built to Brown Corpus as the representative of the first generation of large electronic corpus, corpus linguistics rapid development in the next 40 years, the 20th century 1990s corpus linguistics most rapid period of development. Comparative studies in language and translation studies scholars realize that parallel corpus comparative study of the two languages, translation teaching and research (including automatic translation), bilingual lexicography and translation and foreign language teaching and other aspects were of great value.

Some universities in Canada, Britain, Norway and other Western countries began to establish the first parallel corpus, while the British and French Canadian Hansard Parallel Corpus is the first built. The corpus collected a total of 5000 million words of official records in Canada during the 1973-1986 parliamentary session. The British Academy of Science and Technology, University of Manchester (UMIST) Translation Research Center in 1995 created the world's first translation corpus (Translational English Corpus), mainly collected from various languages into English translation of the text, there are tens of millions of word corpus ( The goal is 50 million words), including novels, biographies, newspapers and periodicals four sub-libraries. University of Oslo English / Norwegian Parallel Corpus (ENPC: English-Norwegian Parallel Corpus) mainly included the source text and the translation, fiction and non-fiction texts. From 46 sub-corpus constituted ECIMC corpus (European CorpusInitiative Multilingual Corpus) covering Europe and other major languages in 27 languages, vocabulary size reached 98 million words, code labels are used in 
SGML language [2]

China's mainland and Hong Kong and Taiwan, there are many universities begin developing parallel corpus, such as the Open University of Hong Kong established for the English and Chinese learning English - Chinese, Chinese - English parallel corpus trilingual, Hong Kong Polytechnic University established Chinese law online corpus, Chinese Language Education Research Center at Beijing Foreign Studies University, developed the 3000 million words / word of English parallel corpus and the like.

The Role of Parallel Corpus. Parallel Corpus comparative study of language is not only used, but also widely used, including automatic translation, including translation teaching, as well as the compilation of bilingual dictionaries; parallel corpus building and research on behalf of the current means of corpus language teaching, the development of language and translation studies compare trend. Researchers parallel corpus can translate text on a comparison of the two words, sentences and stylistic differences, such as the difference between the original and translated text length and the corresponding level of two words in the text. University of Perugia, Italy (University ofPerugia) translation researcher Federico Zanettin dedicated to the author prove parallel corpus to help improve the ability of students to understand the original language and the target language with smoother for translation. One purpose of the new model of language teaching is to cultivate students' self-learning ability, while parallel corpus based translation teaching, to a certain extent, help to help students develop this ability and opportunity to provide personalized, which is bound to further promote the reform of translation teaching promoting explore new translation teaching mode.

\section{The Application of Parallel Corpus in Translation Teaching}

Parallel Corpus corpus with rich resources can not only provide convenience for translation teaching, translation researchers can also provide a more effective reference tools and research platform. Parallel corpus application in Translation Teaching is mainly reflected in the following three aspects: vocabulary, sentence and discourse[3]

The Words Translation Based on Parallel Corpus. Dictionary can only provide a static definition and grammar laws of the word, we can not provide the actual word in the context of dynamic usage. Learners can take advantage of the context of parallel corpus vocabulary translation provided by the practice, by observing a lot of real context, and to find a more appropriate translation. One of the most typical application is the parallel corpus "of bilingual education." Parallel Corpus traditional text incomparable advantages in corpus resources and instantaneous retrieval speed, you can retrieve the translation of a particular expression, provide more examples, make the translation more accurate and authentic; at the same time can also provide a variety of translations for reference, help students enrich translate words, feelings of translation context.

One relationship most of the English words and Chinese words do not exist. In the dictionary, a word often multiple meanings, which means there are a variety of appropriate translation. For example "overcome" the Chinese word for search terms to search in the North outside the common English parallel corpus, the search to 24 using the "overcome" when using the English translation 15 overcome, when students use the English expression "overcome" means, it will not only use the word overcome, but will consider selecting a sample from a rich corpus more consistent with its context words or phrases. The traditional dictionary can not provide similar training, especially by means of Translation Concordancing Chinese words corresponding to the English translation. Visible parallel corpus richer than bilingual dictionary illustration, more real, not only can help students find the right words for the translation, but also help students improve their English language sense, especially when performed English Translation. Given the width of Chinese and English words have different characteristics, Chinese-English translation process, if the English word meaning wider than the Chinese word, the translation often choose hypernyms; while Chinese word meaning wider than when the English word, it would take in a deep understanding of the basis of the original Select the appropriate hyponym, which is translated in the word difficulty level. But through case retrieval corpus, students will be able to intuitively recognize that if a word can have 
multiple translations original word, should choose the best translation of the word in the full understanding of the original foundation.

The Sentences Translation Based on Parallel Corpus. Sentence - aligned parallel corpus in terms of original and the translation is quite neat, using its research conducted sentences translated converter is the most ideal. Translation conversion involves not only the words and rhetorical, covering a variety of sentence conversion, such as the existence of the sentence, not the main sentence, passive sentences, the words omitted sentences and so on[4]

Since the official use of passive sentences written in English, so the text can appear serious and formal, and less Written Chinese seem to use such a structure. Here's to passive sentences to illustrate the benefits of parallel corpus of translation studies.

English sentence:

It has been noted with concern that the stock of books in the library has been declining alarmingly. Students are asked to remind themselves of the rules for the borrowing and return of books, and to bear in mind the needs of other students. Penalties for overdue book will in the future be strictly enforced.

Chinese sentence:

Recently discovered in the library collection strikingly reduced, so the students remember special borrowing requirements specified in the letter, and consider borrowing needs of other students. Where next for overdue books, it will be strictly buckle fines.

English and Chinese, there are some special patterns and grammatical structures, which resulted in confusion when Translation of the students do not know how to deal with, and teaching and cannot explain this phenomenon, then you can take advantage of parallel corpus to clarify learning doubts. For example in the use of parallel corpora of Chinese were translated word structure retrieval of translation can be found, the word is usually translated presented three forms of structure, so that the structure can increase students' perceptions of translation, through a similar exercise for translation retrieval, students deepen their translation of the word structure of intuitive feelings, the teacher then be summarized and interpreted by a suitable method, we can achieve better teaching results.

The Discourse Style and Stylistic Translation Based on Parallel Corpus. The words and sentences of the translation process does not exist in isolation but interrelated and mutual restraint, in order to achieve certain communicative intention according to a certain format combined organic discourse, and therefore in the use of parallel translation corpus to establish clear Text consciousness. Parallel Corpus is also available in its original language translation feature information of the two texts, such as the size of the redundancy of language, vocabulary, co-occurrence and extent of regulation, the characteristics of syntactic pattern and words, so as to further understanding of the stylistic features of language translation and translators style. Does not appear before the parallel corpus, the study of translation of stylistic translation researchers mostly based on experience, can now help of corpus corpus resource rich and powerful retrieval speed, quantitative method to study lengthy translated body styles, such as quantitative analysis of sentence length, individuals preferred language translator term frequency, types of sentences, phrases and narrative structure with forms of expression, in order to further explore the translation style characteristics translator [5].

World Translation Corpus expert, Professor Mona Baker University of Manchester by translating English Corpus of English translator Peter Clark and Peter Bush translated several novels and biographies for a special investigation, and their stylistic translation of the text were compared. Bush originally translated by the three authors, respectively, with native Spanish, South American Spanish and Portuguese written in three languages. Clark translation of the original 40-year-old male from writers and nearly 90 Syrian writer, they are two different styles of Arab writers. Baker comparative analysis were from parts of speech / labeling ratio, average sentence length, and three levels of narrative structure were both asked the following conclusions: Bush asked Mr Clark is more than simple and clear.

Domestic scholars on the "Dream of Red Mansions" chapter back catalog English translations of 
Corpus quantify Statistics found that: - David Hawkes Dream of Red Mansions translation (hereinafter referred to as Hawkes) than yi and Gladys Red Mansions translation (hereinafter referred to as the Yangs) 16 more words. 4\%, more academic vocabulary $1.51 \%$, more unconventional vocabulary $1.186 \%$, Hawkes used unconventional vocabulary frequency (46.455 percent) than "Dream of Red Mansions" Chinese original (46.042\% ); and the Yangs are using unconventional vocabulary frequency (45.269\%) was lower than the "Dream of Red Mansions" Chinese original (46.042\%), pursuant to draw Hawkes is more quaint than the Yangs conclusions

\section{Conclusions}

Translation teaching research based on parallel corpus has both quantitative empirical analysis of in both and qualitative features explanation and it provides the basis for the realization of scientific and standardized of translation curriculum structure, which is conducive to development and deepening of translation studies, facilitate translation practice, improve the quality of translation and translation teaching. From the above words, sentences and chapters three levels, this paper fully explained that if the parallel corpus and search tools being used in translation teaching, students can deepen the level of understanding and awareness the original and the translation chapter to help students achieve multi-channel translation conversion, and continuously improve students translation capabilities.

\section{References}

[1] Guohong: Foreign Language, Vol. 6 (2004) No 53, p.25-26

[2] Jiang Xiaoping: Guangzhou University, Vol. 12 (2005) No 27, p.74-76

[3] Liu Zequan: Chinese Language, Vol. 1 (2006) No 33, p.11-14

[4] Ke Fei: Foreign Language Teaching, Vol. 3 (2007) No33, p.121-124

[5] Liao Qiyi: Foreign Language Teaching and Research, Vol. 3 (2007) No5 p.29-33 